Mongolian Academy of Sciences
Mongolian Journal of Chemistry
Institute of Chemistry \& Chemical Technology

\title{
Coumarins from Peucedanum hystrix growing in Mongolia
}

\author{
Ganbaatar J. ${ }^{1}$, Shults E. E. ${ }^{2}$, Otgonsuren D. ${ }^{1},{ }^{1}$ Radnaeva L.D. ${ }^{3}$, Taraskin $B^{3}$., \\ Badamkhand D. ${ }^{1}$
}
${ }^{1}$ Institute of Chemistry and Chemical Technology, MAS;
${ }^{2}$ Novosibirsk Institute of Organic Chemistry, SB RAS
${ }^{3}$ Baikal Institute of Natural Management SB RAS

\begin{abstract}
It is proved to be that of genus Peucedanum hystrix serve as a source of biologically valuable natural coumarins. Nine angular furocoumarins belonging to class of 2 '-substituted and 2',3'-disubstituted 2',3'-dihydrofurocoumarins have been isolated and structurally isolated. For peucenidin data of X-ray analysis is obtained.
\end{abstract}

Keywords: Peucedanum hystrix, coumarins, oroselol, columbianetin, X-ray analysis.

\section{Introduction}

Dlants of Umbelliferae family are known to be a good source of naturally occurring coumarins for producing of potential medicinal preparations [1]. Coumarins are considered as phytoalexins since plants produce them as defence substances when wounded or attacked by other organisms. Coumarins can be suggested to be beneficial for the plants themselves as natural biocontrolling antipathogenic compounds and for humans as remedy for hyperproliferative skin deseases and as reference compounds in various bioactive tests. Furthermore, coumarin containing plants are valuable as dietary supplements on the basis of their mild antimicrobial and antiinflammatory effects. Coumarins are also active in plant metabolism, taking part in growth regulation.

Plants of genus Peucedanum sp. attract attention of scientists as source of coumarins. Peucedanum hystrix Bge. widely spread in Southern Siberia and Mongolia [2]. To our knowledge a systematic phytochemical investigation of this plant has not been properly carried out. Previously oroselon (1), oroselol (2) and 2'-(S)-Osenecioyl)-2',3'-dihydrooroselol (libanorin) (3) have been isolated from the Peucedanum hystrix Bge. [3].

\section{Experimental}

NMR spectra of compounds were obtained in $\mathrm{CDCl}_{3}$ or $\mathrm{CD}_{3} \mathrm{OD}$ obtained on spectrometers Bruker AV-300 [working frequency $300.13\left({ }^{1} \mathrm{H}\right)$ and $\left.75.47 \mathrm{MHz}\left({ }^{13} \mathrm{C}\right)\right]$ and AV-600 [600.30 $\left({ }^{1} \mathrm{H}\right)$ и $150.96 \mathrm{MHz}$ $\left.\left({ }^{13} \mathrm{C}\right)\right]$. Different types of proton-proton and carbon-proton shifting correlation spectroscopy (COSY, COXH, COLOC, NOESY) were used for assignment of signals in NMR spectra. Multiplesity of signals in ${ }^{13} \mathrm{C}$ NMR spectra determined in regime $J$ modulation. High resolution massspectrometer DFS Thermo Scientific (energy 
of ionizing electrons $70 \mathrm{eW}$, temperature of the evaporator $230-280^{\circ} \mathrm{C}$ ) was used for recording mass-spectra, determining of molecular weight and elemental composition. Melting point determined on table Stuart SMF-38. Specific rotation $[\alpha]_{D}^{20}$ measured on polarimeter PolAAr3005.

X-ray analysis was carried out by $\omega-\varphi$ scanning (width of frames $0.5^{\circ}$ ) on difractometer KAPPA APEX II (Bruker) with doublecoordinate CCD detector by using of MoK $\alpha$ radiation $(\lambda=0.71073 \AA)$.

Purified fractions were investigated by the method of chromato-mass-spectrometry on gas chromatograph Hewlett-Packard 5890/II MSD with quadrupol massspectrometer (HP MSD 5971) as detector. 30 meter quartz column HP-5MS (copolimer of $5 \%$ diphenyl-95\% dimethylenesiloxane) with internal diameter $0.25 \mathrm{~mm}$ and film width of motionless phase $0.25 \mu \mathrm{M}$ were used; temperature $50-280^{\circ} \mathrm{C} 4^{\circ}$ per min, 15 min $280^{\circ} \mathrm{C}$. The content of each compounds have been calculated by the area of GC peak without use of correcting factors and expressed in percentage.

Column chromatography on silica gel (firm Acros, 0.035-0.070 mm) (eluent chloroform-ethanol) was used for isolation individual compounds. The purity of isolated compounds was checked thin layer chromatography method on plate Silufol UV254, used system: chloroform - ethanol, 10:1; petroleum ether - ether, 4:1.

\section{Fraction and Fractionation.}

Air-dried, chopped roots and aerial parts of Peucedanum hystrix Bge. $\left(\begin{array}{lll}500 & \Gamma\end{array}\right)$ exhaustively extracted with $96 \%$ ethanol at room temperature. Alcoholic extract was concentrated until aqueous remainder, diluted by water in the ratio (1:1) and filtrated. Filtrate subjected to fractionation by solvents with increasing polarity: hexane, chloroform, diethyl ether and ethyl acetate, respectively. Triple extraction with solvents $(3 \times 100 \mathrm{ml})$ by heating at reflux was carried out. Fractions were concentrated at rotatory evaporator. Yield of extractive compounds presented at table 1. Further each fractions were separated by column chromatography on silica gel.
Separation of the hexane fraction of the aerial parts of Peucedanum hystrix Bge. $0.9 \mathrm{~g}$ of the hexane fraction was chromatographed by column chromatography, consistently collected fractions. $144 \mathrm{mg}$ eugenin 4, $54 \mathrm{mg}$ umbelliferon $\mathbf{5}, 27 \mathrm{mg}$ oroselon 1, $27 \mathrm{mg}$ columbianetin $\mathbf{6}, 63 \mathrm{mg}$ columbianadin 7, $54 \mathrm{mg}$ peucenidinи $\mathbf{8}$ and $45 \mathrm{mg}$ libanotin 9 were isolated by crystallization from diethylether.

Separation of the hexane fraction of the root of Peucedanum hystrix Bge. $0.8 \mathrm{~g}$ of hexane extract was chromatographed by column chromatography. $64 \mathrm{mg}$ libanorin 3, $88 \mathrm{mg}$ columbianetin $\mathbf{6}, 72 \mathrm{mg}$ columbianadin 7, $72 \mathrm{mg}$ peucenidin $\mathbf{8}, 67 \mathrm{mg}$ libanotin $\mathbf{9}, 16$ $\mathrm{mg}$ furocoumarin $\mathbf{1 0}$ and $20 \mathrm{M \Gamma}$ furocoumarin 11 were isolated.

Separation of the chloroform fraction of the aerial parts of Peucedanum hystrix Bge. $0.7 \mathrm{~g}$ of chloroform extract was chromatographed by column chromatography. $70 \mathrm{mg}$ umbelliferon $\mathbf{5}, 42 \mathrm{mg}$ scopoletin 12, 21 мг oroselon $1,35 \mathrm{mg}$ libanorin $\mathbf{3}, 35 \mathrm{mg}$ columbianetin $\mathbf{6}, 30 \mathrm{mg}$ columbianadin 7, 42 mg peucenidin 8 and $63 \mathrm{mg}$ libanotin 9 were isolated by crystallization from diethylether.

Separation of the chloroform fraction of the roots of Peucedanum hystrix Bge. $0.8 \mathrm{~g}$ of chloroform extract was chromatographed by column chromatography. $16 \mathrm{mg}$ angelicin 13, $48 \mathrm{mg}$ oroselol 2, $24 \mathrm{mg}$ coumarin 14, $24 \mathrm{mg}$ columbianetin $\mathbf{6}, 16 \mathrm{mg}$ coumarin 11, $64 \mathrm{mg}$ umbelliferon 5 and 60 $\mathrm{mg}$ vaginidiol 15 were isolated.

Separation of the ether fraction of the aerial parts of Peucedanum hystrix Bge. $0.96 \mathrm{~g}$ of ether extract was chromatographed by column chromatography. $40 \mathrm{mg}$ oroselol $\mathbf{2}, 30 \mathrm{mg}$ columbianetin $\mathbf{6}, 20$ $\mathrm{mg}$ libanorin $\mathbf{3}, 30 \mathrm{mg}$ peucenidin $3,116 \mathrm{mg}$ vanilic acid 16 and $20 \mathrm{mg}$ trand-ferulic acid 17 were isolated by crystallization from diethylether.

Separation of the ether extract of the roots of Peucedanum hystrix Bge. 0.77 $\mathrm{g}$ of the chloroform extract was chromatographed by column chromatography. $92 \mathrm{mg}$ mixture of coumarins $\mathbf{3}, \mathbf{7}, \mathbf{1 0}$ and $\mathbf{1 1}$, $30 \mathrm{mg}$ vaginidiol 15, $60 \mathrm{mg}$ umbelliferon $\mathbf{5}$ and 69 mglibanotin 9 were isolated. 
Figure 1. Spatial structure of peucenidin (8) molecule

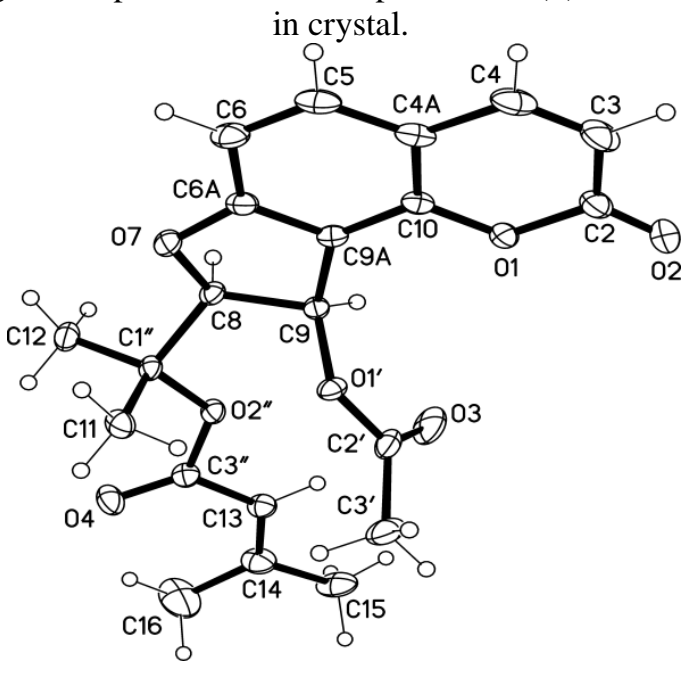

\section{Results and Discussion}

We have investigated extractive compounds from Peucedanum hystrix Bge. collected from the place named Batkhaan uul, Uvurkhangai province. Concentrated ethanolic extract of the aerial parts and roots successively fractionated with hexane, chloroform, diethyl ether and ethyl acetate. The yield of extractive compounds and total content of coumarins in fractions after evaporation of hexane, chloroform and diethyl ether have shown at table 1. Total content of coumarins in the aerial parts and roots were 3.9 and $4.6 \%$. The highest content of coumarins indicated in hexane and chloroform fractions. Extractive compounds separated by column chromatography on silica gel.

From the hexane fraction 5-hydroxy7-methoxy-2-methyl chromon (eugenin) (4) (16\% from extract weight) and coumarins: umbelliferon (5) (6\% from extract weight), oroselon 1 (3\%), 2',3'-dihydrooroselol (columbianetin) (6) (3\%), 2'-O-angeloyl-2',3'dihydrooroselol (sosimin, columbianadin) (7) (7\%), (2'S,3'R)-3'-acetoxy-2'-O-senecioyl2',3'-dihydrooroselol (peucenidin) (8) (6\%) and $(2 ' S, 3 ' R)$ - 2'-O-angeloyl-3'-acetoxy-2',3'dihydrooroselol (libanotin, edultin) (9) (5\%) have been isolated. Oroselon 1 and (2'S)-2methylbutanoyl-2',3'-dihydrooroselol have been identified by spectral data. From the hexane fraction libanorin $\mathbf{3}$ (8\%), columbianetin $6(11 \%)$, columbianadin 7 (9\%), peucenidin $8(9 \%)$ and libanotin 9 (7\%) have been isolated, respectively.
Moreover the compound $\mathbf{1 0}(1.2 \%$ from fraction's weight) and (2'S,3'R)-2'-O-acetyl3'-isobuteryloxy-2',3'-dihydrooroselol (content 2.2\% from fraction's weight).

Chromatographing of chloroformic fraction of ethanolic extract of the aerial parts of Peucedanum hystrix Bge. allow us to concentrate and isolate the main quantity of umbelliferon

5 (10\% from extract's weight) and scopoletin (12) $(6 \%$ from extract's weight), also to isolate oroselon $1(3 \%)$, columbianetin $\mathbf{6}$ $(5 \%)$, libanorin $3(7 \%)$, columbianadin 7 (4\%), peucenidin $8(6 \%)$ and libanotin 9 $(9 \%)$. From chloroformic fraction of ethanolic extract of roots angelicin (13) (2\%), oroselol $2(6 \%)$, columbianetin $6(8 \%)$, libanorin 3 (4\%), 2'S-isovaleryl-2',3'-dihydrooroselol (14) (3\%), columbianadin 7 (3\%), furocoumarin 11 (2\%), umbelliferon $5(8 \%)$, as well as (3'R)-hydroxy-2' $S, 3^{\prime} R$-dihydrooroselol (vaginidiol) (15) (8\%) consequtively isolated.

Subsequent treatment of alcoholic extract of the aerial parts of Peucedanum hystrix Bge. with diethyl ether allow us to isolate additional quantity of oroselol 2 (4\% from extract's weight), columbianetin $\mathbf{6}(3 \%)$, libanorin $3(2 \%)$, peucenidin $8(3 \%)$, vanilic acid (16) (yield 12\%, content 23\%) and transferulic acid (17) (yield 2\%, content $7 \%$ ). From ether fraction of ethanolic extract consecutively isolated vanilic acid 16 (8\%), derivatives of 2',3'-dihydrooroselol $\mathbf{3}, \mathbf{7}, \mathbf{1 0}$ and 11 ( 12\%), vaginidiol (15) (4\%), umbelliferon $\mathbf{5}(8 \%)$, libanotin $9(9 \%)$.

From data presented at table 1 we see that content of coumarins in ethyl acetate fractions was minimum.

Table 1. Yield of extractive compounds and total coumarins of Peucedanum hystrix Bge.

\begin{tabular}{|c|c|c|c|c|c|}
\hline \multirow{2}{*}{ № } & $\begin{array}{c}\text { Solvent } \\
\text { for } \\
\text { extraction }\end{array}$ & $\begin{array}{r}\text { Aerial parts } \\
\text { Yield of } \\
\text { extractive } \\
\text { compoun } \\
\text { ds, \% }\end{array}$ & $\begin{array}{c}\text { Total } \\
\text { content of } \\
\text { coumarins, } \\
\%\end{array}$ & $\begin{array}{c}\text { Yield of } \\
\text { extractive } \\
\text { compoun } \\
\text { ds, \% }\end{array}$ & $\begin{array}{c}\text { Total } \\
\text { content of } \\
\text { coumarins, } \\
\%\end{array}$ \\
\hline 1 & Hexane & 1.8 & 1.25 & 2.4 & 1.5 \\
\hline 2 & $\begin{array}{c}\text { Chlorofor } \\
\text { m }\end{array}$ & 3.4 & 2.1 & 3.0 & 2.4 \\
\hline 3 & $\begin{array}{c}\text { Diethyl } \\
\text { ether }\end{array}$ & 2.5 & 0.45 & 1.8 & 0.5 \\
\hline 4 & $\begin{array}{c}\text { Ethylacet } \\
\text { ate }\end{array}$ & 2.2 & 0.1 & 2.5 & 0.2 \\
\hline
\end{tabular}




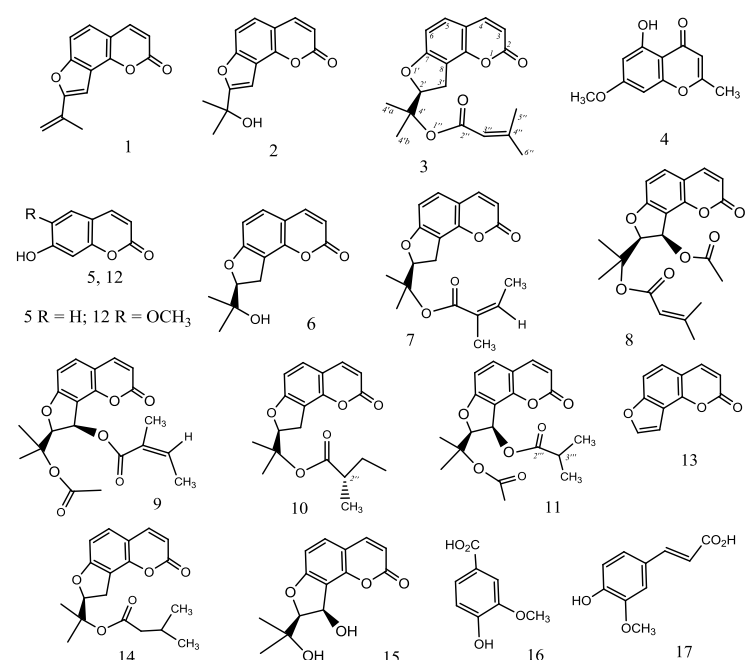

Figure 2. Isolated and identified compounds.

Structures of compounds have been established on the basis of their phusical, chemical and spectral characteristics, as well as comparison with literature data. For peucenidin $8 \mathrm{X}$-ray analysiswas done (Figure 2).

Geometric parameters of molecule of compound $\mathbf{8}$ have been proved by statistical data [4]. In Cambridge base of structural data [5] found seven identical structures with 2',3'dihydrofurocoumarin skeleton including (2'S,3'R)-3'-senecoyl-2-O-acetyl-2',3'-

dihydrooroselol (isopeucenidin) [6]. However in $\mathrm{CBCD}$ are absent 3D coordinates for isopeucenidin therefore we could not do comparisonal analysis of geometrical parameters of isopeucenidin with peucenidin 8. In molecule of compound $\mathbf{8}$ observed intramolecular hydrogen bonds of $\mathrm{C}-\mathrm{H}$... $\mathrm{O}$ type which suggested to be formed due to presence of angeloyl and acetyl substituents (table 2).

Table 2. Parameters of intramolecular and intermolecular hydrogen bonds in the crystal of the compound $\mathbf{8}$.

\begin{tabular}{|c|c|c|c|c|c|c|}
\hline № & $\mathrm{D}-\mathrm{H} \ldots \mathrm{A}$ & $\mathrm{D}-\mathrm{H}, \AA$ & $\mathrm{H} \ldots \mathrm{A}, \AA$ & $\mathrm{D} \ldots \mathrm{A}, \AA$ & $\begin{array}{c}\text { Ungle } \\
\text { D-H-A, }{ }^{\circ}\end{array}$ & $\begin{array}{c}\text { Operationof symme- } \\
\text { try }\end{array}$ \\
\hline 1. & $\mathrm{C} 9-\mathrm{H} 9 \ldots 03$ & 1.00 & 2.28 & $2.688(2)$ & 103 & - \\
2. & $\mathrm{C} 11-\mathrm{H} 11 \mathrm{~A} \ldots 01$ & 0.98 & 2.33 & $3.008(3)$ & 125 & - \\
3. & $\mathrm{C} 11-\mathrm{H} 11 \mathrm{C} \ldots 04$ & 0.98 & 2.55 & $3.088(2)$ & 115 & - \\
4. & $\mathrm{C} 12-\mathrm{H} 12 \mathrm{C} \ldots .04$ & 0.98 & 2.39 & $2.928(2)$ & 114 & - \\
5. & $\mathrm{C} 16-\mathrm{H} 16 \mathrm{C} \ldots 04$ & 0.98 & 2.18 & $2.942(3)$ & 134 & - \\
6. & $\mathrm{C} 3-\mathrm{H} 3 \ldots 03$ & 0.95 & 2.29 & $3.157(3)$ & 152 & $3-\mathrm{x},-0.5+\mathrm{y}, 0.5-\mathrm{z}$ \\
7. & $\mathrm{C} 8-\mathrm{H} 8 \ldots 02$ & 1.00 & 2.52 & $3.470(2)$ & 160 & $-1+\mathrm{x}, \mathrm{y}, \mathrm{z}$ \\
\hline
\end{tabular}

The investigation showed that Peucedanum hystrix Bge. represents as a reliable source of furocoumarins. In addition to oroselol 2 three groups of dihydrofurocoumarins: Monoethers $8(S)-8,9-$ dihydrooroselol $3,7,8,10,14$, vaginidio 15 and their 2,3-diethers $\mathbf{8}, \mathbf{9}, \mathbf{1 1}$ have been isolated. 2'-Substituted and 2',3'-disubstituted $2^{\prime}, 3^{\prime}$-dihydrofurocoumarins attract attention in connection with known antitumour activity. Thus, 2'(S)-columbianetin sulphate possess antiproliferative activity [7]. Dihydrofurocoumarins vaginidiol 15 [6, 8] and angelmarin [9] also have antitumour activity.

Thus, we have obtained new data about metabolites composition of the plant Peucedanum hystrix Bge. It is proved to be that this plant might serve as source of $2^{\prime}$ substituted and 2',3'-disubstituted 2',3'dihydrofurocoumarins. Deserve attention isolation from Peucedanum hystrix Bge. highly distributed in plants chromone 4 [11] as well as vanilic $\mathbf{1 6}$ and trans-ferulic $\mathbf{1 7}$ acid.

\section{Spectral data of individual compounds}

Spectral and analytical data for umbelliferon 5 and scopoletin 12 correspond to literature data [12]. ${ }^{13} \mathrm{C}$ NMR spectral data of furocoumarins shown at table 3 .

Oroselon $\{8$-(prop-1-ene-2-yl)-2H-furo[2,3h]chromen-2-one $\}$ (1). M. p. $177-180^{\circ} \mathrm{C}$ (from ethylacetate). In paper [2] m.p. 179$180^{\circ} \mathrm{C}$. IR spectra (V, см -1): 760, 812, 831, 907, 1020, 1123, 1154, 1374, 1553, 1617, 1680. ${ }^{1} \mathrm{H} \mathrm{NMR}$ spectra $\left(\mathrm{CDCl}_{3}, \delta, \mathrm{ppm}\right.$, $\mathrm{J} / \mathrm{Hz}): 2.13\left(3 \mathrm{H}, \mathrm{s}, \mathrm{CH}_{3}\right), 5.24\left(1 \mathrm{H}, \mathrm{s}, \mathrm{H}-5^{\prime}\right)$, $5.82\left(1 \mathrm{H}, \mathrm{s}, \mathrm{H}-5^{\prime}\right), 6.36(1 \mathrm{H}, \mathrm{d}, J=9.6, \mathrm{H}-3)$, $6.96(1 \mathrm{H}, \mathrm{s}, \mathrm{H}-3$ ') $, 7.30,7.34$ (both $\mathrm{d}, 1 \mathrm{H}, \mathrm{J}=$ 8.5, H-5,6), $7.77(1 \mathrm{H}, \mathrm{d}, J=9.6, \mathrm{H}-4)$. Massspectra, m/z (I $\left.I_{\text {comp }}, \%\right): 226$ (100), 198 (82), 183 (48), 171 (24), 155 (53), 141 (28), 115 (43), 101 (12), 75 (26), 63 (78). Found, \%: C 74.18; $\mathrm{H}$ 4.26. $\mathrm{C}_{14} \mathrm{H}_{10} \mathrm{O}_{3}$. Calculated, \%: $\mathrm{C}$ 74.33; H 4.46.

Oroselol \{8-(2-hydroxypropane-2-yl)-2Hfuro[2,3- $\boldsymbol{h}]$ chromen-2-one $\}$ (2). M.p. 150$154^{\circ} \mathrm{C}$ (from ether). In paper [2] m.p. 149$151{ }^{\circ} \mathrm{C} .{ }^{1} \mathrm{H}$ NMR spectra $\left(\mathrm{CDCl}_{3}, \delta\right.$, ppm, $\mathrm{J} / \mathrm{Hz}): 1.74\left(\mathrm{~s}, 6 \mathrm{H}, 2 \times \mathrm{CH}_{3}\right.$ at $\left.\mathrm{C}-4{ }^{\prime}\right), 6.35(1 \mathrm{H}$, d, $J=9.8, \mathrm{H}-3), 6.98(1 \mathrm{H}, \mathrm{s}, \mathrm{H}-3$ ') $7.32(1 \mathrm{H}$, д, $J=8.5, \mathrm{H}-6), 7.37(1 \mathrm{H}, \mathrm{d}, J=8.5, \mathrm{H}-5)$, $7.78(1 \mathrm{H}, \mathrm{d}, J=9.8, \mathrm{H}-4)$. Mass-spectra, $\mathrm{m} / \mathrm{z}$ 
(I $\left.I_{\text {comp }}, \%\right): 224$ (32), 229 (100), 212 (5), 201 (15), 187 (8), 171 (14), 115 (15), 101 (18), 43 (23). Found: $[M]$ 224.2034. $\mathrm{C}_{14} \mathrm{H}_{12} \mathrm{O}_{4}$. Calculated: 244.2035.

\section{Libanorin $\{8(S)$-(2-senecioxypropane-2-yl)-} 8,9-dihydro-2H-furo[2,3- $h]$-chromen-2-

one\} (3). M.p. $75-77^{\circ} \mathrm{C}$ (from ether). $[\alpha]_{\mathrm{D}}=$ +188.3 (c 0.88, $\mathrm{CHCl}_{3}$ ). In paper [13] m.p. $79^{\circ} \mathrm{C},[\alpha]_{\mathrm{D}}=+197\left(\mathrm{c} 2.2, \mathrm{CHCl}_{3}\right)$. UV spectra (ethanol), $\lambda_{\max } / \mathrm{nm} \quad(\lg \varepsilon): 206 \quad(4.11), 237$ (3.68), 298 (2.77), 329 (4.13). IR spectra, (v, $\left.\mathrm{cm}^{-1}\right)$ : 806, 832, 930, 980, 1024, 1069, 1263, $1385,1460,1506,1620,1720,1736,3090 .{ }^{1} \mathrm{H}$ NMR spectra $\left(\mathrm{CDCl}_{3}, \delta, \mathrm{ppm}, \mathrm{J} / \mathrm{Hz}\right): 1.53$, 1.61 (both s, $3 \mathrm{H}, \mathrm{CH}_{3}$ at $\mathrm{C}-4$ ), $1.86,2.10$ (both s, $3 \mathrm{H}, \mathrm{CH}_{3}$ at $\mathrm{C}-5$ "), $3.36\left(2 \mathrm{H}, \mathrm{m}, \mathrm{H}-3^{\prime}\right)$, $5.21(1 \mathrm{H}, \mathrm{dd}, J=7.6,8.2, \mathrm{H}-2 \mathrm{\prime}), 5.58(1 \mathrm{H}$, broad s, H-4"), $6.23(1 \mathrm{H}, \mathrm{d}, J=9.6, \mathrm{H}-3)$, $6.80(1 \mathrm{H}, \mathrm{d}, J=8.4, \mathrm{H}-6), 7.27(1 \mathrm{H}, \mathrm{d}, J=$ 8.4, H-5), $7.62(1 \mathrm{H}, \mathrm{d}, J=9.6, \mathrm{H}-4)$. Massspectra, m/z (I $\left.I_{\text {comp }}, \%\right): 328$ (10), 303 (10), 286 (5), 244 (16), 243 (11), 229 (42), 227 (15), 201 (8), 187 (16), 83 (100), 43 (25). Found: $[M]$ 328.1314. $\mathrm{C}_{19} \mathrm{H}_{20} \mathrm{O}_{5}$. Calculated: 328.1311. $5.58(1 \mathrm{H}$, broad s, H-4"), $6.23(1 \mathrm{H}, \mathrm{d}$, $J=9.6, \mathrm{H}-3), 6.80(1 \mathrm{H}, \mathrm{d}, J=8.4, \mathrm{H}-6), 7.27(1 \mathrm{H}$, $\mathrm{d}, J=8.4, \mathrm{H}-5), 7.62(1 \mathrm{H}, \mathrm{d}, J=9.6, \mathrm{H}-4)$. Massspectra, m/z (I $\left.I_{\text {comp }}, \%\right): 328(10), 303(10), 286$ (5), 244 (16), 243 (11), 229 (42), 227 (15), 201 (8), 187 (16), 83 (100), 43 (25). Found: [M] 328.1314. $\mathrm{C}_{19} \mathrm{H}_{20} \mathrm{O}_{5}$. Calculated: 328.1311.

Table 4.

\begin{tabular}{|c|c|c|c|c|c|c|c|c|c|c|c|c|}
\hline Atom ${ }^{*}$ & 1 & 2 & 3 & 6 & 7 & 8 & 9 & 10 & 11 & 13 & 14 & 15 \\
\hline C. -2 & 160.62 & 160.69 & 160.76 & 160.91 & 160.72 & 159.70 & 159,51 & 160.55 & 159.81 & 160.66 & 160.28 & 160.47 \\
\hline C.3 & 113.88 & 113.95 & 111.91 & 112.07 & 111.86 & 112.96 & 113.21 & 112.05 & 113.08 & 113.82 & 113.02 & 112.55 \\
\hline$C-4$ & 14.32 & 144.36 & 143.78 & 143.83 & 143.75 & 143.35 & 143.41 & 143.88 & 143.53 & 144.35 & 144.01 & 143.85 \\
\hline $\mathrm{CHa}_{\mathrm{a}}$ & 118.29 & 113.42 & 113.24 & 113.88 & 112.88 & 113.15 & 113.24 & 113.31 & 113.27 & 116.75 & 113.21 & 113.10 \\
\hline C.5 & 123.79 & 123.41 & 128.21 & 128.61 & 128.43 & 131.22 & 131.21 & 127.12 & 131.32 & 124.66 & 127.24 & 130.77 \\
\hline C.6 & 108.21 & 108.48 & 106.48 & 106.53 & 106.41 & 107.46 & 107.60 & 106.43 & 107.03 & 108.64 & 106.56 & 107.95 \\
\hline C.7 & 156.90 & 156.91 & 163.70 & 163.56 & 163.71 & 163.48 & 163.39 & 163.05 & 163.52 & 157.19 & 163.18 & 163.50 \\
\hline C.8 & 132.18 & 117.41 & 112.75 & 112.96 & 112.96 & 112.42 & 112.94 & 112.88 & 112.85 & 113.35 & 112.91 & 116.35 \\
\hline$C .8 a$ & 148.08 & 148.12 & 151.05 & 151.14 & 151.06 & 151.56 & 151.56 & 152.20 & 151.82 & 148.33 & 152.35 & 151.52 \\
\hline C.2. & 157.94 & 164.22 & 88.84 & 91.12 & 89.05 & 88.46 & 88.32 & 88.68 & 88.19 & 145.72 & 88.59 & 90.97 \\
\hline$C .3^{\prime}$ & 99.59 & 97.37 & 27.37 & 27.40 & 27.35 & 68.36 & 68.11 & 27.12 & 68.33 & 103.92 & 27.21 & 69.98 \\
\hline$C A^{\prime}$ & 113.40 & 69.11 & 81.04 & 71.63 & 81.56 & 80.13 & 81.02 & 81.79 & 78.26 & . & 81.93 & 72.15 \\
\hline $\begin{array}{l}\mathrm{CH}_{3} \\
\text { חpr }\end{array}$ & 19.06 & 28.58 & 21.45 & 24,06 & 22.46 & 22.19 & 23.30 & 21.18 & 22.07 & . & 21.26 & 26.33 \\
\hline$(C-4)$ & & 28.58 & 23.08 & 26.10 & 25.48 & 25.10 & 25.20 & 23.46 & 25.28 & - & 22.34 & 27.49 \\
\hline
\end{tabular}

* Used numeration of coumarin sceleton's atoms like in structure (3) (scheme 1). Note: *For compound

1: $114.46\left(\mathrm{C}=\right.$ at $\left.\mathrm{C}-4^{\prime}\right)$;

3: 165.61 (C-2"), 116.77 (C-3"), 156.27 (C-4"), 22.03 (C-5"), 21.88 (C-6");

7: 167.02 (C-2"), 128.11 (C-3"), 137.31 (C-4"), 20.26 (C-5"), 15.67 (C-6");
8: 165.82 (C-2"), 116.41 (C-3"), 156.89 (C-4"), 19.93 (C-5"), $20.75(\mathrm{C}-6 "), 168.46\left(\mathrm{COCH}_{3}\right), 27.28\left(\mathrm{CH}_{3}\right)$; 9: 166.08 (C-2"), 128.46 (C-3"), 137.71 (C-4"), 15.62 (C-5"), 20.81 (C-6"), $170.51\left(\mathrm{COCH}_{3}\right), 22.32\left(\mathrm{CH}_{3}\right)$; 10: 174.62 (C-2"), 44.21 (C-3"), 26.75 (C-4"), 16.23 $\left(\mathrm{CH}_{3}\right), 11.31\left(\mathrm{CH}_{3}\right)$;

11: $11.15\left(\mathrm{CH}_{3}\right), 15.58\left(\mathrm{CH}_{3}\right), 22.15\left(\mathrm{CH}_{3}\right), 42.18$ (C3"'), $170.41\left(\mathrm{COCH}_{3}\right), 174.62$ c $(\mathrm{C}-2$ "');

14: $12.65\left(\mathrm{CH}_{3}\right), 16.29\left(\mathrm{CH}_{3}\right), 28.46(\mathrm{C}-3 "), 42.08(\mathrm{C}-$ $4 "), 173.33$ (ㄷ-2").

Eugenin (5-hydroxy-2-methyl-7-methoxy4H-chromen-4-one) (4), oil. ${ }^{1} \mathrm{H}$ NMR spectra $\left(\mathrm{CDCl}_{3}, \delta, \mathrm{ppm}, \mathrm{J} / \mathrm{Hz}\right): 2.42\left(3 \mathrm{H}, \mathrm{s}, \mathrm{CH}_{3}\right)$, $3.85\left(3 \mathrm{H}, \mathrm{s}, \mathrm{OCH}_{3}\right), 6.03(1 \mathrm{H}, \mathrm{s}, \mathrm{H}-3), 6.29$ $(1 \mathrm{H}, \mathrm{d}, \mathrm{J}=2.0, \mathrm{H}-6), 6.35(1 \mathrm{H}, \mathrm{d}, \mathrm{J}=2.0, \mathrm{H}-$ 8), $12.67(1 \mathrm{H}, \mathrm{s}, \mathrm{OH}) \cdot{ }^{13} \mathrm{C}$ NMR spectra $\left(\mathrm{CDCl}_{3}, \delta, \mathrm{ppm}, \mathrm{J} / \mathrm{Hz}\right): 20.59\left(\mathrm{CH}_{3}\right), 55.75$ $\left(\mathrm{OCH}_{3}\right), 92.32(\mathrm{C}-8), 98.22(\mathrm{C}-6), 105.24(\mathrm{C}-$ 4a), 108.65 (C-3), 158.18 (C-8a), 162.54 (C5), 165.41 (C-7), 166.49 (C-2), 182.44 (C-4). Mass-spectra, m/z (I comp, \%): 206 (100), 189 (35), 177 (20). Found: [M] 206.0579. $\mathrm{C}_{11} \mathrm{H}_{10} \mathrm{O}_{4}$. Calculated: 206.0571.

Columbianetin $\{8(S)$-(2-hydroxypropane-2yl)-8,9-dihyhydro- $2 H$-furo $[2,3-h]$-chromen2-one (6). M.p. $160-163^{\circ} \mathrm{C}$ (from ether). $[\alpha]_{\mathrm{D}}$ $=+195.36$ (c 1.2, $\left.\mathrm{CHCl}_{3}\right)$. In paper [7] m.p. $163^{\circ} \mathrm{C},[\alpha]_{\mathrm{D}}=+264\left(\mathrm{c} 1.66, \mathrm{CH}_{3} \mathrm{OH}\right) .{ }^{1} \mathrm{H}$ NMR spectra $\left(\mathrm{CDCl}_{3}, \delta, \mathrm{ppm}, \mathrm{J} / \mathrm{Hz}\right): 1.22$, 1.35 (both s, $3 \mathrm{H}, \mathrm{CH}_{3}$ at $\mathrm{C}-4$ '), $3.30(2 \mathrm{H}, \mathrm{m}$, H-3'), $4.77\left(1 \mathrm{H}, \mathrm{t}, J=8.6, \mathrm{H}-2^{\prime}\right), 6.18(1 \mathrm{H}, \mathrm{d}$, $J=9.6, \mathrm{H}-3), 6.73(1 \mathrm{H}, \mathrm{d}, J=8.2, \mathrm{H}-6), 7.26$ $(1 \mathrm{H}, \mathrm{d}, J=8.2, \mathrm{H}-5), 7.60(1 \mathrm{H}, \mathrm{d}, J=9.6, \mathrm{H}-$ 4), $9.50 \mathrm{~s}(1 \mathrm{H}, \mathrm{OH})$. Mass-spectra, m/z (I $I_{\text {comp }}$, $\%): 246$ (56), 228 (10), 213 (14), 203 (10), 187 (100), 175 (25), 160 (48), 131 (21), 103 (12), 77 (22), 59 (69). Found: [M] 246.0264. $\mathrm{C}_{14} \mathrm{H}_{14} \mathrm{O}_{4}$. Calculated: 246.0269.

\section{Columbianadin} $\{8(S)-(2-$ angeloyloxypropane-2-yl) -8,9-dihydro- $2 \mathrm{H}$ furo[2,3- $h]$-chromen-2-one $\}$ (7). M.p. 117$120^{\circ} \mathrm{C}$ (from ether). $[\alpha]_{\mathrm{D}}=+225.6$ (c 1.8, $\mathrm{CHCl}_{3}$ ). In paper [14] m.p. $118.5-119^{\circ} \mathrm{C}$, $[\alpha]_{\mathrm{D}}=+227\left(\mathrm{c} 2.8, \mathrm{CHCl}_{3}\right) .{ }^{1} \mathrm{H}$ NMR spectra $\left(\mathrm{CDCl}_{3}, \delta, \mathrm{ppm}, \mathrm{J} / \mathrm{Hz}\right): 1.62\left(3 \mathrm{H}, \mathrm{s}, \mathrm{CH}_{3}\right.$ at $\mathrm{C}-$ 3"), 1.63 (both s, $3 \mathrm{H}, \mathrm{CH}_{3}$ at $\mathrm{C}-4$ '), $1.85(3 \mathrm{H}$, s, $\mathrm{CH}_{3}$ at C-4"), $3.36\left(2 \mathrm{H}, \mathrm{m}, \mathrm{H}-3^{\prime}\right), 5.18(1 \mathrm{H}$, $\left.\mathrm{dd}, J=7.8,8.2, \mathrm{H}-2^{\prime}\right), 5.98(1 \mathrm{H}, \mathrm{m}, \mathrm{H}-4 ")$, $6.20(1 \mathrm{H}, \mathrm{d}, J=9.8, \mathrm{H}-3), 6.68(1 \mathrm{H}, \mathrm{d}, J=$ $8.2, \mathrm{H}-6), 7.22(1 \mathrm{H}, \mathrm{d}, J=8.2, \mathrm{H}-5), 7.60$ $(1 \mathrm{H}, \mathrm{d}, J=9.6, \mathrm{H}-4)$. Mass-spectra, $\mathrm{m} / \mathrm{z}$ 
$\left(I_{\text {comp }}, \%\right): 328$ (10), 270 (8), 257 (6), 246 (5), 228 (35), 213 (100), 187 (18), 131 (16), 83 (16), 55 (42). Found: $[M] 328.1314$. $\mathrm{C}_{19} \mathrm{H}_{20} \mathrm{O}_{5}$. Calculated: 328.1311 .

\section{Peucenidin $\quad\{(8 S, 9 R)-9-A c e t o x y-8-(2-$} senecioyloxypropane-2-yl)-8,9-dihydro-2Hfuro[2,3-h]chromen-2-one $\}$ (8). M.p. 122$125^{\circ} \mathrm{C}$ (from ethylacetate). $[\alpha]_{\mathrm{D}}=-48.2(\mathrm{c}$ $\left.1.23, \mathrm{CHCl}_{3}\right)$. In papers $[13,15][\alpha]_{\mathrm{D}}=-46^{\circ}$. UV spectra (ethanol), $\lambda_{\max } / \mathrm{nm}$ (lge): 207 (5.12), 226 (4.12), 298 (3.77), 322 (4.68). IR spectra, $\left(v, \mathrm{~cm}^{-1}\right): 806,848,862,938,980$, $1005,1024,1053,1075,1114,1132,1320$, $1354,1490,1576,1620,1647,1727,1752$, 3093. ${ }^{1} \mathrm{H}$ NMR spectra $\left(\mathrm{CDCl}_{3}, \delta, \mathrm{ppm}\right.$, $\mathrm{J} / \mathrm{Hz}$ ): $1.61,1.70$ (both s, $3 \mathrm{H}, \mathrm{CH}_{3}$ at $\mathrm{C}-4$ '), 1.86, 2.11 (both s, 3H, $\mathrm{CH}_{3}$ at C-5"), 2.02 $\left(3 \mathrm{H}, \mathrm{s}, \mathrm{C}_{3} \mathrm{C}=\mathrm{O}\right), 5.15\left(1 \mathrm{H}, \mathrm{d}, J=7.0, \mathrm{H}-2^{\prime}\right)$, $5.58(1 \mathrm{H}$, broad s, H-4"), $6.21(1 \mathrm{H}, \mathrm{d}, J=9.6$, $\mathrm{H}-3), 6.82(1 \mathrm{H}, \mathrm{d}, J=8.5, \mathrm{H}-6), 6.97(1 \mathrm{H}, \mathrm{d}$, $\left.J=7.0, \mathrm{H}^{\prime} 3^{\prime}\right), 7.40(1 \mathrm{H}, \mathrm{d}, J=8.5, \mathrm{H}-5), 7.61$ $(1 \mathrm{H}, \mathrm{d}, J=9.6, \mathrm{H}-4)$.Mass-spectra, $\mathrm{m} / \mathrm{z}\left(I_{\text {comp }}\right.$, \%): 386 (15), 326 (12), 311 (13), 303 (8), 286 (22), 261 (9), 244 (28), 243 (15), 229 (40), 227 (32), 201 (24), 187 (22), 83 (100), 43 (25). Found: $[M]$ 386.1364. Calculated: 386.1360. Found, \%: C 65,38; H 5,32. $\mathrm{C}_{21} \mathrm{H}_{22} \mathrm{O}_{7}$. Calculated, \%: C 65.28; H 5.74.

\section{Libanotin (edultin) $\quad\left\{\left(8 S, 9^{\prime} R\right)-9\right.$ - (angeloyloxypropane-2-yl)-8-acetoxy-8,9- dihydro- $2 H$-furo[2,3- $h$ ]chromen-2-one $\}$ (9)} M.p. $154-156^{\circ} \mathrm{C}$ (from hexane). $[\alpha]_{\mathrm{D}}=+78.6$ (c $1.3, \mathrm{CHCl}_{3}$ ). In paper [6] т.пл. $155-157^{\circ} \mathrm{C}$, $[\alpha]_{\mathrm{D}}=+79.5\left(\mathrm{c} 0.83, \mathrm{CHCl}_{3}\right)$. UV spectra (ethanol), $\lambda_{\max } / \mathrm{nm}$ (lge): 207 (5.12), 232 (4.21), 300 (3.36), 318 (4.21). ${ }^{1} \mathrm{H}$ NMR spectra $\left(\mathrm{CDCl}_{3}, \delta, \mathrm{ppm}, \mathrm{J} / \mathrm{Hz}\right): 1.59,1.71$ (both s, 3H, $\mathrm{CH}_{3}$ at $\mathrm{C}-4$ ), $1.81,1.92$ (both s, $3 \mathrm{H}, \mathrm{CH}_{3}$ at C-2"' and C-3"'), $2.01(3 \mathrm{H}, \mathrm{s}$, $\left.\mathrm{C}_{3} \mathrm{C}=\mathrm{O}\right), 5.28\left(1 \mathrm{H}, \mathrm{d}, J=7.0, \mathrm{H}-2^{\prime}\right), 6.08$ (1H, m, H-3"'), 6.23 (1H, d, $J=9.6, \mathrm{H}-3)$, $6.85(1 \mathrm{H}, \mathrm{d}, J=8.5, \mathrm{H}-6), 7.02(1 \mathrm{H}, \mathrm{d}, J=$ 7.0, H-3'), $7.41(1 \mathrm{H}, \mathrm{d}, J=8.5, \mathrm{H}-5), 7.62$ $(1 \mathrm{H}, \mathrm{d}, J=9.6, \mathrm{H}-4)$. Mass-spectra, $\mathrm{m} / \mathrm{z}$ (I comp,$\%): 386$ (18), 311 (12), 287 (12), 261 (8), 243 (32), 227 (18), 187 (73), 83 (100), 43 (54). Found: $[M]$ 386.1368. Calculated: 386.1360 .
\{(8S,11S)-[2-(2-methylbutanoyl) oxypropane-2-yl)]-8,9-dihydro- $2 H$ furo[2,3- $h]$-chromen-2-one (10). M.p. 85$88^{\circ} \mathrm{C}$ (from ether). $[\alpha]_{\mathrm{D}}=+192.6$ (c 1.1, $\mathrm{CHCl}_{3}$ ). In paper [7] m.p. $88-91^{\circ} \mathrm{C},[\alpha]_{\mathrm{D}}=$ +194 (c 0.67, $\mathrm{CHCl}_{3}$ ). ${ }^{1} \mathrm{H}$ NMR spectra $\left(\mathrm{CDCl}_{3}, \delta, \mathrm{ppm}, \mathrm{J} / \mathrm{Hz}\right): 0.85(3 \mathrm{H}, \mathrm{t}, J=7.0$, $\mathrm{CH}_{3}$ at $\left.\mathrm{C}-4 "\right), 1.03\left(3 \mathrm{H}, \mathrm{d}, J=7.0, \mathrm{CH}_{3}\right.$ at $\mathrm{C}$ $3^{\prime \prime)}, 1.30,1.45$ (1H, м, $\left.J=7.0, \mathrm{H}-4 "\right), 1.50$, 1.56 (both s, 3H, $\mathrm{CH}_{3}$ at $\mathrm{C}-4$ '), $3.28(1 \mathrm{H}, \mathrm{m}$, $\left.J_{\text {gem }}=15.7, \mathrm{H}^{\prime} 3^{\prime}\right), 3.36\left(1 \mathrm{H}, \mathrm{m}, J_{\text {gem }}=15.7\right.$, H-3'), $5.08\left(1 \mathrm{H}, \mathrm{dd}, J=7.6,9.4, \mathrm{H}-2^{\prime}\right), 6.18$ $(1 \mathrm{H}, \mathrm{d}, J=9.6, \mathrm{H}-3), 6.71(1 \mathrm{H}, \mathrm{d}, J=8.2, \mathrm{H}-$ 6), $7.22(1 \mathrm{H}, \mathrm{d}, J=8.2, \mathrm{H}-5), 7.60(1 \mathrm{H}, \mathrm{d}, J$ $=9.6, \mathrm{H}-4)$. Mass-spectra, $\mathrm{m} / \mathrm{z}\left(I_{\text {comp }}, \%\right): 330$ (16), 305 (8), 259 (6), 231 (30), 213 (100), 187 (31), 176 (28), 85 (32), 57 (46). Found: [M] 330.3459. $\quad \mathrm{C}_{19} \mathrm{H}_{22} \mathrm{O}_{5}$. Calculated: 330.3467 .

$\{(8 S, 9 R)-8-(2-A c e t o x y p r o p a n e-2-y l)-9-(2-$ methyl)propanoyloxy)-8,9-dihydro-2Hfuro[2,3- $\boldsymbol{h}]$ chromen-2-one (11) $\}$. M.p. 153$156^{\circ} \mathrm{C}$ (from ether). $[\alpha]_{\mathrm{D}}=+100.2$ (c 0.6, $\mathrm{CHCl}_{3}$ ). In paper [8] m.p. $154-156^{\circ} \mathrm{C},[\alpha]_{\mathrm{D}}=$ +97.9 (c 0.19, $\mathrm{CHCl}_{3}$ ). ${ }^{1} \mathrm{H}$ NMR spectra $\left(\mathrm{CDCl}_{3}, \delta, \mathrm{ppm}, \mathrm{J} / \mathrm{Hz}\right): 1.17(3 \mathrm{H}, \mathrm{d}, J=6.8$, $\mathrm{CH}_{3}$ at $\left.\mathrm{C}-3{ }^{\prime \prime}\right), 1.19\left(3 \mathrm{H}, \mathrm{d}, J=7.0, \mathrm{CH}_{3}\right.$ at $\mathrm{C}$ $\left.3^{\prime \prime \prime}\right), 1.59,1.75$ (both $\mathrm{s}, 3 \mathrm{H}, \mathrm{CH}_{3}$ at $\left.\mathrm{C}-4^{\prime}\right), 2.05$ $\left(3 \mathrm{H}, \mathrm{s}, \mathrm{CH}_{3} \mathrm{C}=\mathrm{O}\right), 2.53\left(1 \mathrm{H}, \mathrm{m}, \mathrm{H}-3^{\prime \prime \prime}\right), 5.28$ $\left(1 \mathrm{H}, \mathrm{d}, J=6.7, \mathrm{H}-2^{\prime}\right), 6.20(1 \mathrm{H}, \mathrm{d}, J=9.6, \mathrm{H}-$ 3), $6.84(1 \mathrm{H}, \mathrm{d}, J=8.5, \mathrm{H}-6), 7.00(1 \mathrm{H}, \mathrm{d}, J=$ $6.7, \mathrm{H}-3$ ') $7.42(1 \mathrm{H}, \mathrm{d}, J=8.5, \mathrm{H}-5), 7.62$ $(1 \mathrm{H}, \mathrm{d}, J=9.6, \mathrm{H}-4)$. Mass-spectra, $\mathrm{m} / \mathrm{z}$ $\left(I_{\text {comp }}, \%\right)$ : 374 (15), 314 (8), 299 (12), 286 (15), 261 (18), 244 (33), 229 (100), 213 (22), 187 (38), 158 (20), 71 (54), 43 (61). Found: $[M]$ 374.2257. $\mathrm{C}_{19} \mathrm{H}_{22} \mathrm{O}_{5}$. Calculated: 374.2259 .

Angelicin (2H-furo[2,3- $h]$ chromen-2-one) (13). M. p. $133-136^{\circ} \mathrm{C}$ (from ether). In paper [16] m.p. $136-137^{\circ} \mathrm{C}$. UV spectra (ethanol), $\lambda_{\max } / \mathrm{nm}$ (lge): 251 (4.12), 302 (3.85), 326 (4.04). ${ }^{1} \mathrm{H}$ NMR spectra $\left(\mathrm{CDCl}_{3}, \delta, \mathrm{ppm}\right.$, $\mathrm{J} / \mathrm{Hz}): 6.36(1 \mathrm{H}, \mathrm{d}, J=9.6, \mathrm{H}-3), 7.10(1 \mathrm{H}$, $\left.\mathrm{dd}, J=2.0,1.6, \mathrm{H}-3^{\prime}\right), 7.34(1 \mathrm{H}, \mathrm{d}, J=8.5, \mathrm{H}-$ 5), $7.41(1 \mathrm{H}, \mathrm{dd}, J=8.5,1.6, \mathrm{H}-5), 7.68(1 \mathrm{H}$, $\left.\mathrm{d}, J=2.0, \mathrm{H}-2^{\prime}\right), 7.79(1 \mathrm{H}, \mathrm{d}, J=9.6, \mathrm{H}-4)$. Mass-spectra, m/z (I $\left.I_{\text {comp }}, \%\right): 186$ (100), 158 (88), 149 (27), 130 (14), 102 (28). Found: [M] 186.0312. $\mathrm{C}_{11} \mathrm{H}_{6} \mathrm{O}_{3}$. Calculated: 186.0317. 
\{8S-(2-isovaleryl)oxypropane-2-yl)]-8,9dihudro-2 $H$-furo[2,3- $h]$-chromen-2-one $\}$ (14). M.p. $72-74^{\circ} \mathrm{C}$ (from ether). $[\alpha]_{\mathrm{D}}=$ +302.5 (c 1.1, $\mathrm{CHCl}_{3}$ ). In paper [13] т.пл. $76-77^{\circ} \mathrm{C},[\alpha]_{\mathrm{D}}=+305\left(\right.$ c $\left.0.4, \mathrm{CH}_{3} \mathrm{OH}\right) .{ }^{1} \mathrm{H}$ NMR spectra $\left(\mathrm{CDCl}_{3}, \delta, \mathrm{ppm}, \mathrm{J} / \mathrm{Hz}\right): 0.91$ $\left(3 \mathrm{H}, \mathrm{t}, J=7.1, \mathrm{CH}_{3}\right.$ at $\left.\mathrm{C}-4 "\right), 1.16(3 \mathrm{H}, \mathrm{d}, J=$ 7.0, $\mathrm{CH}_{3}$ at $\left.\mathrm{C}-4 "\right), 1.48,1.62(1 \mathrm{H}, \mathrm{m}, \mathrm{H}-3 ")$, $1.60,1.68$ (both $\mathrm{s}, 1 \mathrm{H}, \mathrm{CH}_{3}$ at $\mathrm{C}-4$ '), $2.36 \mathrm{~m}$ $(1 \mathrm{H}, \mathrm{H}-4 "), 3.30\left(1 \mathrm{H}, \mathrm{m}, J_{\text {gem }}=15.9, \mathrm{H}-3\right.$ '), $\left.3.36\left(1 \mathrm{H}, \mathrm{m}, J_{\mathrm{gem}}=15.9, \mathrm{H}-3\right)^{\prime}\right), 5.06(1 \mathrm{H}, \mathrm{dd}$, $\left.J=7.8,9.6, \mathrm{H}-2^{\prime}\right), 6.19(1 \mathrm{H}, \mathrm{d}, J=9.6, \mathrm{H}-3)$, $6.72(1 \mathrm{H}, \mathrm{d}, J=8.4, \mathrm{H}-6), 7.22(1 \mathrm{H}, \mathrm{d}, J=$ 8.4, H-5), $7.61(1 \mathrm{H}, \mathrm{d}, J=9.6, \mathrm{H}-4)$. Massspectra, $\mathrm{m} / \mathrm{z}\left(I_{\text {comp }}, \%\right): 330$ (10), 281 (5), 228 (30), 213 (100), 187 (28), 176 (20), 159 (11), 131 (18), 85 (25), 57 (31). Found: [M] 330.3461. $\mathrm{C}_{19} \mathrm{H}_{22} \mathrm{O}_{5}$. Calculated: 330.3467 .

\section{Vaginidiol $\quad\{(8 S, 9 R)-9-H y d r o x y-8-(2-$ hydroxypropane-2-yl)-8,9-dihydro-2H-}

furo[2,3-h]chromen-2-one (15) \}. M.p. 170$173^{\circ} \mathrm{C}$ (from ethanol). $[\alpha]_{\mathrm{D}}=+224.2(c$ 1.2, $\mathrm{CHCl}_{3}$ ). In paper [17] m.p. $168-169^{\circ} \mathrm{C},[\alpha]_{\mathrm{D}}$ $=+231(\mathrm{c} 0.01$, EtOH $) .{ }^{1} \mathrm{H}$ NMR spectra $\left(\mathrm{CDCl}_{3}, \delta, \mathrm{ppm}, \mathrm{J} / \mathrm{Hz}\right): 1.48,1.55$ (both s, 3H, $\mathrm{CH}_{3}$ at $\left.\mathrm{C}-4^{\prime}\right), 4.20(1 \mathrm{H}$, broad $\mathrm{s}, 1 \mathrm{H}, \mathrm{OH})$, $4.35\left(1 \mathrm{H}, \mathrm{d}, J=5.6, \mathrm{H}^{2}\right), 5.77(1 \mathrm{H}, \mathrm{d}, J=$ 5.6, H-3'), $6.20(1 \mathrm{H}, \mathrm{d}, J=9.6, \mathrm{H}-3), 6.82$ $(1 \mathrm{H}, \mathrm{d}, J=8.2, \mathrm{H}-6), 7.35(1 \mathrm{H}, \mathrm{d}, J=8.2, \mathrm{H}-$ 5), $7.63(1 \mathrm{H}, \mathrm{d}, J=9.6, \mathrm{H}-4)$. Mass-spectra, $\mathrm{m} / \mathrm{z}\left(I_{\text {comp }}, \%\right): 262$ (30), 229 (19), 213 (15), 203 (19), 191 (27), 188 (22), 187 (50), 186 (100), 158 (62), 149 (27), 134 (22), 131 (32), 129 (17), 102 (34), 89 (19), 59 (61). Found: $[M] \quad 262.0832 . \quad \mathrm{C}_{14} \mathrm{H}_{14} \mathrm{O}$. Calculated: 262.0836 .

4-Hydroxy-3-methoxybenzoic acid (vanilic acid) (16), m.p. $210-213{ }^{\circ} \mathrm{C}$ (from ether) (in paper [18], 213-214 $\left.{ }^{\circ} \mathrm{C}\right) .{ }^{1} \mathrm{H}$ NMR spectra $\left(\mathrm{CDCl}_{3}, \delta, \mathrm{ppm}, \mathrm{J} / \mathrm{Hz}\right): 3.92\left(3 \mathrm{H}, \mathrm{s}, \mathrm{OCH}_{3}\right)$, $6.84(1 \mathrm{H}, \mathrm{d}, \mathrm{J}=8.3, \mathrm{H}-5), 7.59(1 \mathrm{H}, \mathrm{dd}, \mathrm{J}=$ 8.3 and $1.9, \mathrm{H}-6), 7.55(1 \mathrm{H}, \mathrm{d}, \mathrm{J}=1.9, \mathrm{H}-2)$. ${ }^{13} \mathrm{C}$ NMR spectra $\left(\mathrm{CDCl}_{3}, \delta, \mathrm{ppm}, \mathrm{J} / \mathrm{Hz}\right)$ : $56.31\left(\mathrm{OCH}_{3}\right), 113.51$ (C-2), 115.47 (C-5), 122.69 (C-4), 125.02 (C-6), 148.11 (C-3), $152.01(\mathrm{C}-1), 169.81(\mathrm{C}=\mathrm{O})$. Mass-spectra, $\mathrm{m} / \mathrm{z}\left(I_{\text {comp }}, \%\right): 168$ (100), 153 (65), 125 (31), 97 (50), 77 (10).
trans-Ferulic acid (17), m.p. $165-167{ }^{\circ} \mathrm{C}$ (from ether) (in paper [19], 168-169 ${ }^{\circ} \mathrm{C}$ ). ${ }^{1} \mathrm{H}$ NMR spectra $\left(\mathrm{CDCl}_{3}, \delta, \mathrm{ppm}, \mathrm{J} / \mathrm{Hz}\right): 3.92$ $\left(3 \mathrm{H}, \mathrm{s}, \mathrm{OCH}_{3}\right), 6.31(1 \mathrm{H}, \mathrm{d}, \mathrm{J}=16.1, \mathrm{H}-\alpha)$, $6.82(1 \mathrm{H}, \mathrm{d}, \mathrm{J}=8.3, \mathrm{H}-5), 6.90(1 \mathrm{H}, \mathrm{dd}, \mathrm{J}=$ 8.3 and $2.0, \mathrm{H}-6), 7.11(1 \mathrm{H}, \mathrm{d}, \mathrm{J}=2.0, \mathrm{H}-2)$, $7.59(1 \mathrm{H}, \mathrm{d}, J=16.1, \mathrm{H}-\beta) .{ }^{13} \mathrm{C}$ NMR spectra $\left(\mathrm{CDCl}_{3}, \delta, \mathrm{ppm}, \mathrm{J} / \mathrm{Hz}\right): 55.82\left(\mathrm{OCH}_{3}\right), 110.05$ (C-2), $115.11(\mathrm{C}-\alpha), 115.24(\mathrm{C}-5), 123.00$ (C6), 129.91 (C-1), $145.71(\mathrm{C}-\beta), 148.50$ (C-4), $150.64(\mathrm{C}-3), 172.91(\mathrm{C}=\mathrm{O})$. Mass-spectra, m/z (I $\left.I_{\text {comp }}, \%\right): 194$ (100), 179 (18), 148 (10), 133 (17), 105 (8), 77 (10).

\section{Conclusions}

1. It is proved to be that plants of genus Peucedanum hystrix might serve as source of biologically valuable natural coumarins.

2. Nine angular furocoumarins belonging to class of 2'-substituted and 2',3'disubstituted 2',3'-dihydrofurocoumarins have been isolated and structurally isolated.

3. For peucenidin data of X-ray analysis is obtained.

\section{References}

1. А. В. Липеева, Э. Э. Шульц, М. М. Шакиров, Г. А. Толстиков, (2011) Журн. орган. химии. (in press).

2. М. Г. Пименов, (1991), Ботан. журн. 76, 1387 Растительные ресурсы России: Дикорастущие цветковые растения, их компонентный состав и биологическая активность. Т. 3. Семейства Fabaceae-Apiaceae. / Отв. Редактор А.Л. Буданцев. (2010) СПб.; М.: Издательство КМК, 601.

3. Л. И. Шагова, Г. А. Кузнецова, Л. П. Маркова, В. М. Виноградова, (1981), Химия природн. соедин., 518.

4. F. H. Allen, O. Kenard, D.G.Watson, L. Bramer, A.G. Orpen, R.Taylor, (1987) J. Chem. Soc., Perkin Trans. 2, S1-19.

5. F. H. Allen, (2002) Acta Cryst., B58, 380.

6. C. Kawasaki, T. Okuyama, S. Shibata, Y. Iitaka, (1984) Planta Med., 50, 492. 
7. Y. A. Kim, C.-S. Kong, S. S. Yea, Y. Seo, (2010) Food Chem. Toxicol., 48, 722.

8. A. Mizuno, M. Takata, Y. Okada, T. Okuyama, H. Nishino, A. Nishino, J. Takayasu, A. Iwashima, (1994) Planta Med., 60, 333.

9. S. Awale, E.M.N. Nakashima, S.K. Kalauni, Y. Tezuka, Y. Kurashima, J. Lu, H. Esumi, S. Kadota, (2006) Bioorg. Med. Chem. Lett., 16, 581.

10. T. Mosmann, J. Immunol. (1983) Methods, 16, 55;

11. W.-L. Chang, L.-W. Chiu, J.-H. Lai, H.C. Lin, (2003) Phytochemistry, 64, 1375.

12. В. М. Маликов, А. И. Саидходжаев, (1998) Химия природн. соедин., 560.

13. F. Bohlmann, M. Grenz, (1969) Chem. Ber., 102, 1673.

14. B. E. Nielsen, J. Lemmich, (1964) Acta Chem. Scand., 18, 1379.

15. Г. И. Захаров, П. Б. Терентьев, Г. К. Николаев, (1972) Химия природн. соедин., 271.
16. T.RS. Seshadri, S. M. Sood, K. L. Handa, Vishwapaul, (1967) Tetrahedron, 23, 1883 .

17. B. D. Gupta, S.K. Benerjee, K. L. Handa, (1976) Phytochemistry, 15, 576.

18. M. Kuroyanagi, S. Fukushima, K. Yoshihira, S. Natori, T. Dechatiwongse, K. Mihashi, M. Nishi, S.Hara, (1980) Chem. Pharm. Bull., 28, 2948.

19. C. J. Kelley, C. Harruff, M. Carmack, (1976) J. Org. Chem. 41, 449.

20. G.M. Sheldrick. (2004) SADABS.Version 2.01.Bruker AXS Inc. Madison, Wisconsin, USA.

21. G.M. Sheldrick. (1997) SHELX-97 Programs for Crystal Structure Analysis (Release 97-2). - Univ. Göttingen, Germany.

22. A. L. Spek, (2003) J. Appl. Crystallogr., 36, 7.

23. I. J. Bruno, J. C. Cole, P. R. Edgington, M. Kessler, C. F. Macrae, P. McCabe, J. Pearson, R. Taylor, Acta Cryst., B58, 389 (2002).

24. J. K.Wilson, J. M. Sargent, A. W. Elgie, J. G. Hill, Taylor C. G., (1990) Br. J. Cancer., 62, 189. 